\title{
Use of IVIVC in the Development of Oral Extended-Release Formulations: A Personal Perspective
}

\author{
Mario A. Gonzalez ${ }^{1, *}$ and Douglas F. Smith ${ }^{2}$ \\ ${ }^{1} P$ 'Kinetics International, Inc., Pembroke Pines, FL \\ ${ }^{2}$ DFS Consulting, LLC, Tucson, AZ
}

\section{INTRODUCTION}

VIVC is the common abbreviation when referring to in vitro-in vivo correlations. This in turn refers to the relationship between an in vivo pharmacokinetic parameter derived from drug plasma concentrations after oral dosing and an in vitro parameter obtained by a dissolution procedure commonly used in the quality control of an oral dosage form. An IVIVC could therefore be a simple relationship between a PK parameter such as $C_{\max }$ and the time for $50 \%$ dissolution of an oral dosage form. Ideally, a change in the dissolution properties should be reflected by a change in the PK parameters. For example, a shorter time for $50 \%$ dissolution should lead to a shorter time to $C_{\max }$. Alternatively, the relationship could be between the in vitro dissolution profile and the in vivo dissolution (in vivo absorption) profile. Obviously, any relationship would be beneficial to formulation scientists, since demonstration of in vivo equivalence of a new drug product or a reformulated current drug product by dissolution testing is preferable to relying on expensive and time-consuming clinical pharmacokinetic studies.

\section{DEVELOPING AN IVIVC}

Ideally, an IVIVC should be predictive such that a change in an in vitro property predicts a change in an in vivo property. This leads to the question as to what should be correlated. Among the critical events that led to attempts to establish an IVIVC was the publication in 1963 by Wagner and Nelson (1) of a mathematical relationship to transform a plasma concentration profile into an absorption profile. The original Wagner-Nelson equation is

$$
\frac{A_{t}}{V}=C_{t}+\beta \int_{0}^{t} C d t
$$

where $A_{t}$ is the amount absorbed at any time, $V$ is drug volume of distribution, $C_{t}$ is the measured plasma concentration at time $t$, and $\beta$ is the apparent rate constant of elimination, which is a hybrid constant calculated from the elimination phase of a semi-log plot of a plasma concentration profile (now frequently represented by $\lambda_{z}$ ).

Using the Wagner-Nelson method, Levy (2) demonstrated in 1965 that it was possible to show a relationship between dissolution/disintegration of aspirin and the absorption profile for different aspirin tablets.

Jumping ahead a few years, in 1983 a linear relationship between the percentage dissolved at a certain time point and the percentage absorbed at that same time point was demonstrated (3). To establish this relationship, a modified version of the Wagner-Nelson equation was utilized:

$$
F_{t}=\frac{(A U C)_{t}+C_{t} / \beta}{(A U C)_{\infty}}
$$

This equation allows the calculation of $F_{t}$ the fraction of the total amount of drug absorbed at a certain time, $t$. This illustrates that for every plasma concentration at a certain time $\left(C_{t}\right)$, it is possible to calculate a value that represents the fraction of the total dose absorbed at that time point. For every time point where a blood sample has been collected, $F_{t}$ can be calculated resulting in a full absorption profile after a single-dose bioavailability study. This calculation thus allows the conversion of a typical plasma concentration profile ( $C_{t}$ vs $t$ ) into an absorption profile labeled as a "Wagner-Nelson plot" in Figure 1.

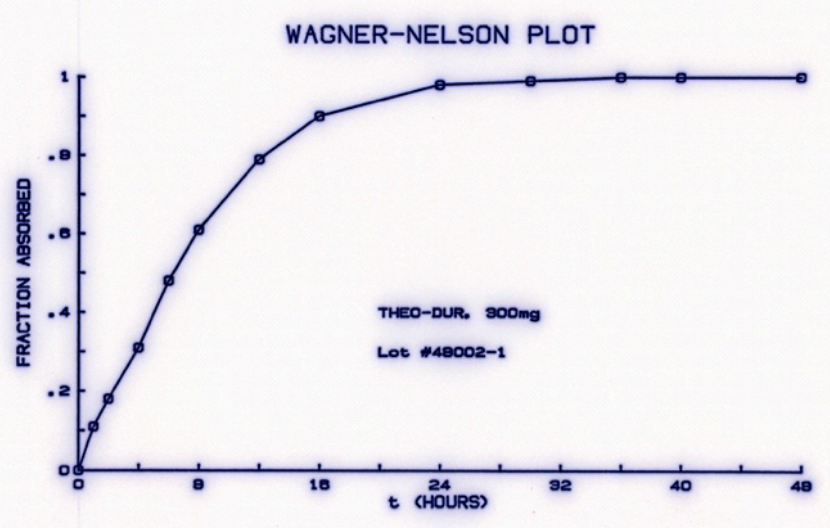

Figure 1. Example of a Wagner-Nelson plot describing the absorption profile for an extended-release theophylline, Theo-Dur.

If an absorption profile can be compared with a dissolution profile, then the quest for an IVIVC may be fulfilled. Figure 2 is a plot of the fraction absorbed at a certain time point on the

${ }^{*}$ Corresponding author. 
$X$-axis versus the fraction absorbed at that same time point on the $Y$-axis. The data used for this figure were obtained using the same lot of extended-release theophylline in both a dissolution test as well as a bioavailability study. Figure 2 illustrates that as the fraction dissolved increases, the fraction absorbed also increases; thus, a true in vitroin vivo relationship was established and the dissolution method could be designated as biorelevant. While Figure 2 demonstrates a linear relationship, linearity is not a necessary requirement.

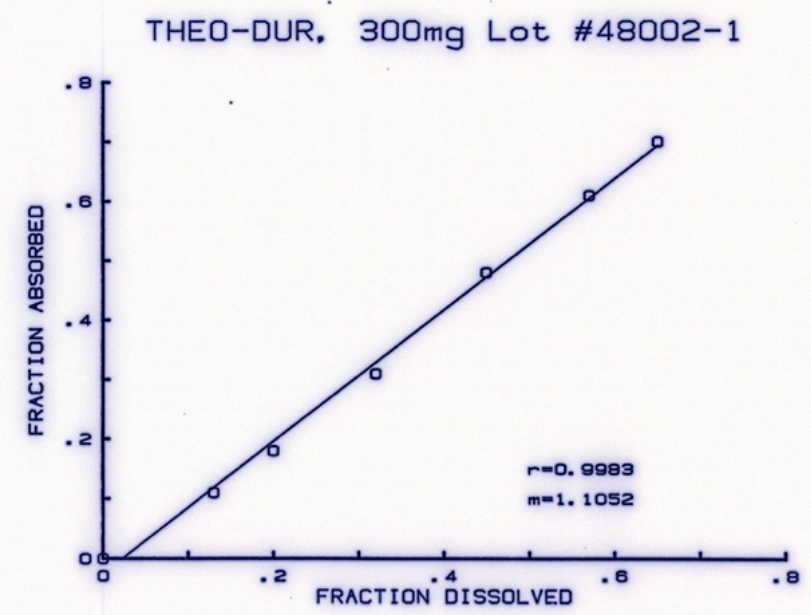

Figure 2. Plot of fraction absorbed versus fraction dissolved for an extended-release theophylline product. An increase in fraction dissolved clearly results in an increase in fraction absorbed. The same lot of product should be used in both the dissolution test and the bioavailability study.

The Wagner-Nelson equation was derived for a drug whose disposition is best described by one exponential term, that is, a drug with one-compartment characteristics. For extended-release formulations of two-compartment drugs, the Wagner-Nelson equation is still applicable. This is because of the lack of an obvious distribution phase due to the slow drug input from an extended-release formulation. For those drugs with obvious two-compartment model traits, the absorption profile should be calculated using the Loo-Riegelman equation (4). The use of this relationship in a simplified form has also been discussed as the Exact Loo-Riegelman equation (5). While the absorption of many drugs can be analyzed using the Wagner-Nelson equation, the Loo-Riegelman equation should be used when the drug has obvious two-compartmental characteristics after oral dosing.

\section{DEFINITIONS OF AN IVIVC}

As mentioned previously, a true IVIVC should be predictive. It should be possible to use in vitro data from a new formulation to predict the in vivo performance of that product. Ideally, a plasma concentration-time profile should be predicted from in vitro dissolution data for an IVIVC to be recognized by regulatory agencies. The FDA officially defined the requirements for an IVIVC in a guidance first issued in 1997 (6). It should be noted, however, that this guidance is a carefully developed document that actually encompasses a time span from the mid-1980s through the late 1990s. The following is a brief summary of the initiatives that led to the FDA guidance on IVIVC:

- AAPS/FDA workshops in late 1980s and early 1990s.

- USP stimuli article in 1987-Levels A, B, and C (7).

- USP <1088> describes IVIVC techniques.

- ER SUPAC Workshop II Report in 1993.

- FDA guidance issued in 1997 for ER dosage forms (6). USP General Chapter <1088 > was the first official IVIVC publication drafted in 1987 by a team of experts under the leadership of a Ciba-Geigy pharmaceutical scientist, Dr. Lewis Leeson, and recently revised (8). In this USP chapter, the concept of IVIVC and what it should constitute was first described. It also introduced the idea of different levels of IVIVC, which was later adopted in the 1997 FDA guidance.

\section{Level A Correlation}

To meet the definition of a Level A correlation, the IVIVC must be a point-to-point relationship between the in vivo parameter (e.g., absorption rate) and the drug's in vitro dissolution. For a Level A correlation, a product's in vitro dissolution curve is compared with its absorption profile, which could be obtained using the WagnerNelson equation or some other deconvolution technique. The in vivo absorption profile should utilize all of the data in the plasma concentration profile resulting from a bioavailability study. Besides the Wagner-Nelson or LooRiegelman methods, a model-independent, mathematical deconvolution technique is acceptable. In some cases, the in vivo and in vitro curves may be superimposable, though this is not a requirement of the FDA guidance. Techniques that may be used to make the curves superimposable are: vary the dissolution method (e.g., by increasing or decreasing the mixing speed), change the dissolution apparatus, or change the dissolution medium. If this is done, the same conditions must be applied for any other formulation to be tested. The guidance also allows the use of a constant offset value of the time scale so that the curves may be superimposed and the equations describing the in vitro and in vivo curves are the same. Alternatively, if the dissolution and absorption curves are different, a mathematical relationship may be developed that relates the two resulting curves in a predictive model for a plasma concentration-time profile using in vitro dissolution data.

The Level A IVIVC is considered the strongest possible demonstration of a relationship between dissolution and bioavailability data. A point-to-point correlation utilizes every plasma level and every dissolution point collected at different time intervals. The IVIVC must exist for different formulations with different dissolution rates, not simply for one product with a specific dissolution profile. If this can be demonstrated, the validity of the IVIVC can be verified by predicting an in vivo plasma profile using the dissolution profile of a different formulation other than the one tested in the original bioavailability study. When a Level A 
correlation has been demonstrated, the in vitro dissolution curve may serve as a surrogate for in vivo performance. Therefore, a change in a formulation manufacturing site, method of manufacture, or other scale-up and postapproval changes (SUPAC) may be justified to regulatory agencies without the need for additional bioavailability/ bioequivalence studies.

While the Level A IVIVC is the most desirable, to demonstrate a valid Level A correlation, it is important that the following criteria are met:

- Only human subjects should be used in the bioavailability studies used to arrive at an IVIVC.

- The in vivo study should utilize a statistically powered number of subjects to adequately characterize the drug product in vivo performance.

- While an IVIVC can be established with only two formulations, it is recommended that three formulations with different release rates be used to establish an IVIVC. These formulations should demonstrate corresponding differences in absorption profiles.

- As recommended by the FDA guidance, the predictability of the Level A IVIVC must be demonstrated by calculating the absolute percent prediction error between an observed pharmacokinetic parameter and the predicted value of that parameter. An average absolute percent prediction error of $10 \%$ or less for $C_{\max }$ and $A U C$ is recommended.

\section{Level B Correlation}

If a point-to-point correlation between the in vivo and in vitro parameters cannot be established, a Level B correlation should be considered. Level B correlations utilize the principles of statistical moment analysis. The mean in vitro dissolution time is compared with either the mean residence time or the mean in vivo dissolution time. As with a Level A correlation, Level B utilizes all of the in vitro and in vivo data but is not a point-to-point correlation. It does not correlate the actual in vivo plasma profiles, but rather a parameter that results from statistical moment analysis of the plasma profile such as mean residence time (MRT). Because there are a number of different plasma profiles (shapes) that will produce similar MRT values, it is not possible to rely upon a Level $B$ correlation to predict a plasma profile from dissolution data. In addition, a Level B correlation cannot be used to justify SUPAC manufacturing changes, but it does help to identify a biorelevant dissolution method for quality control of manufactured drug products.

\section{Level C Correlation}

A Level C IVVC may be the easiest to establish, but as with a Level B IVIVC, it suffers from a lack of predictive capability. This level of IVIVC relates a dissolution value at a certain time point (e.g., $t_{50 \%}, t_{90 \%}$ ) to a single pharmacokinetic parameter such as $A U C, C_{\max }$ or $T_{\max }$. As with a Level $B$ correlation, a Level $C$ correlation is a single- point correlation and does not reflect the complete shape of the plasma profile, which best defines the performance of a modified-release drug formulation. Since this type of correlation is not predictive of actual in vivo product performance, it is generally only useful as a guide in formulation development or as a quality control procedure for manufactured products. Because of its obvious limitations, a Level $\mathrm{C}$ correlation has limited usefulness in predicting in vivo drug performance and is subject to the same limits as a Level B correlation. In the FDA guidance addressing IVIVC (6), it is stated that it may be possible to obtain biowaivers based on a multiple Level $\mathrm{C}$ correlation, that is, that multiple lots would have to be utilized to define the Level $\mathrm{C}$ correlation. The manner in which this correlation could be obtained is defined in the guidance. However, the FDA indicates that if such a correlation is achievable, it is likely that the development of a Level A correlation is also feasible for that particular product. Recently, however, Dr. Patrick Marroum, formerly with the FDA, reported that the agency had approved a Level C IVIVC utilizing eight different lots of a drug formulation (9). He illustrated a successful Level $\mathrm{C}$ correlation with data from the lots that were evaluated in multiple parallel studies rather than the crossover studies advocated in the FDA guidance.

\section{PROS AND CONS OF IVIVCS}

The subject of in vitro-in vivo correlations has increased in popularity as may be judged by the increase in publications and workshops dealing with this topic. An obvious reason is the need for pharmaceutical scientists to validate dissolution methods by establishing that they are predictive of bioavailability; that is, that they are biorelevant. This has long been a desirable objective for any chemist conducting dissolution studies of pharmaceutical products. As previously mentioned, if a Level A correlation exists, the in vitro dissolution test becomes a surrogate for the plasma concentration profile. SUPAC changes can then be supported by dissolution data alone, thus saving months of planning and executing a bioequivalence study comparing an approved formulation with a SUPAC formulation. Furthermore, the dissolution specifications proposed for a new formulation can be supported by use of the IVIVC with dissolution data at the low and high end of the specifications. If the dissolution profiles for the high and low specifications predict in vivo plasma curves that would be bioequivalent on the basis of predicted $C_{\max }$ and $A U C$ values, the dissolution specifications should be approved by a regulatory agency. On the negative side, it can be expensive and time consuming to establish a Level A IVIVC depending on the drug or formulation. For example, a highly variable drug (\%CV intrasubject variability greater than $30 \%$ ) would require considerably more enrolled subjects to ascertain whether observed in vivo differences are due to formulation or subject effects. It may not always be possible to prepare different formulations with different dissolution profiles. If an IVIVC, however, cannot 
be validated, it may behoove the formulation scientist to establish the existence of any in vitro-in vivo relationship (IVIVR) that can still guide formulation development. An IVIVR can still be of great value to a formulation group when the dissolution method is predictive of in vivo behavior. The example illustrated in Figure 2 serves as an example of an IVIVR that can be accomplished with only one lot of a formulation and one single-dose bioavailability study. Figure 2, however, also illustrates an IVIVR that results in a linear correlation between in vitro and in vivo data. This is not a prerequisite for a successful IVIVC or IVIVR.

\section{PROCEDURES TO DEVELOP AND VALIDATE A LEVEL A IVIVC}

The following discussion is not meant to suggest that only the approach below can be used for developing an IVIVC. Any of a number of approaches is possible to arrive at a valid IVIVC or, at a minimum, an IVIVR. The example cited above in the CRS Workshop (9) should remind us all that a guidance does not have to be followed blindly and that good science can still lead to success. What this section offers is one possible procedure for developing a Level A correlation. It should be noted that this discussion was originally presented in the revised USP $<1088>$ (7) and the figures are modified from those used in the USP chapter by the authors.

To perform a successful deconvolution, it is important to be familiar with the pharmacokinetics of the drug itself and how the drug may behave when it is formulated as an extended-release or other modified-release dosage form. For example, if a drug is known to be fully absorbed but demonstrates pre-systemic metabolism, it is best to assume $100 \%$ bioavailability for purposes of calculating an absorption rate. This is because the drug is fully absorbed, but due to a first-pass effect, less of the drug may be seen systemically than if the drug were administered as an immediate-release oral dose. If the extent of absorption relative to an immediate-release or solution dosage form is used to calculate an absorption profile, the input profiles will not be superimposable with the one calculated assuming $100 \%$ absorption. A point-to-point correlation between in vitro and in vivo data may still be achieved regardless of the method used to calculate absorption or input rates.

Different dissolution profiles of formulations should be obtained as illustrated in Figure 3. The formulations should be modified sufficiently to produce different dissolution profiles, yet the formulations should have the same excipients in all the lots to be tested. The formulation modifications utilized in these batches should be based upon factors that would be expected to influence the product dissolution rate and might be the result of common manufacturing variables. In vitro drug release must be obtained using the batches to be used in the bioavailability study. While it is preferable to use official dissolution equipment, any method to be used in future quality control of the product may be used. The dissolution method should be carefully characterized to identify the variables that influence the dissolution. Variables that should be studied include the mixing intensity and dissolution medium including the $\mathrm{pH}$ of the medium, ionic strength, use of surfactants or enzymes, and so forth. The number of variables investigated will depend largely on whether a correlation can be developed with the in vitro results obtained using common dissolution procedures. The resulting dissolution profiles from the use of different dissolution media are illustrated in Figures 3 and 4 in which the same formulations were tested in both water (Figure 3) and an acid buffer (Figure 4).

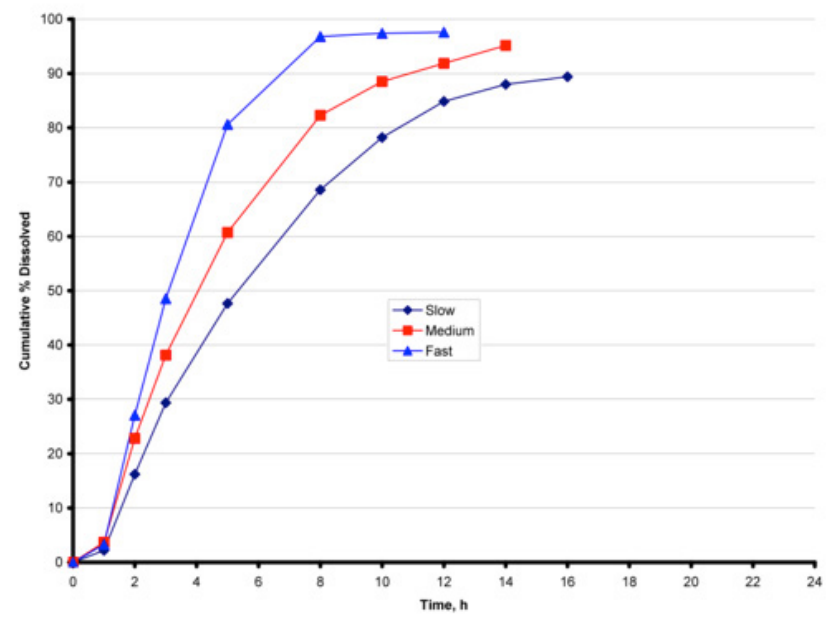

Figure 3. Mean dissolution profiles of a new MR formulation with USP Apparatus 2, 50 rpm, $0.9 \mathrm{~L}$ water at $37^{\circ} \mathrm{C}$.

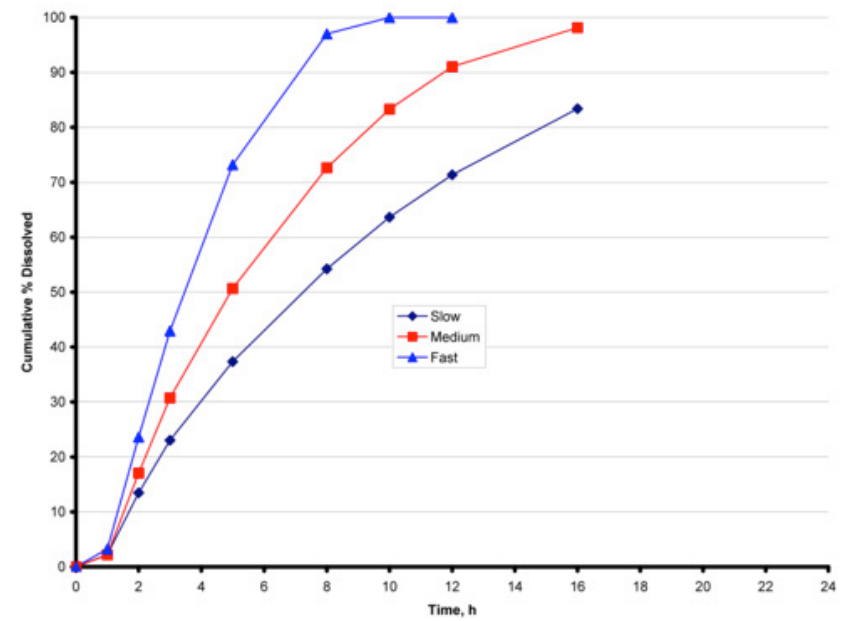

Figure 4. Mean dissolution profiles of an MR Formulation with USP Apparatus 2, $50 \mathrm{rpm}$, $0.9 \mathrm{~L} \mathrm{pH} 4.5$ buffer at $37^{\circ} \mathrm{C}$.

The plasma level profiles (plasma drug concentration-time data) obtained in the bioavailability study of the modified-release dosage form are treated by a deconvolution procedure. The resulting data may represent an absorption profile or simply a drug input rate from the dosage form. This calculation will result in an absorption 
profile that in some cases may be considered an in vivo dissolution profile. The latter interpretation is possible if the rate-controlling step of the dosage form is its dissolution rate. Any deconvolution procedure (for example, the Wagner-Nelson method or mathematical deconvolution) will result in a drug input rate profile. Figure 5 illustrates the results of numerical deconvolution of the plasma profiles obtained for the batches found in Figures 3 and 4.

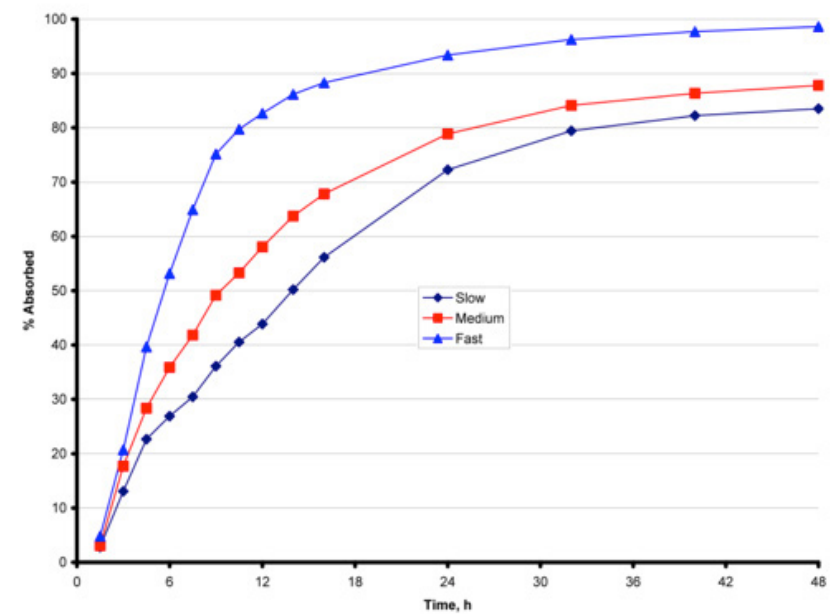

Figure 5. Mean absorption profiles from numerical deconvolution of plasma profiles.

The in vitro dissolution curves should then be compared with the drug input rate curves. This can be performed by various methods. Simply positioning one curve on the other can often indicate the existence of a correlation. These curves may then be quantified by defining an equation for each curve and comparing the corresponding parameters. The simplest way to demonstrate a correlation is to plot the fraction absorbed in vivo versus the fraction released in vitro as illustrated by Figures 6 and 7. With a Level A correlation, this relationship is often linear with a slope approaching 1 , in which case the point-to-point correlation becomes a one-to-one relationship. As may be seen in Figures 6 and 7, however, a linear correlation is not necessary; a meaningful IVIVC may be found to be curvilinear. If there is a delay after dosing before the dosage form begins to release drug in vivo, a lag time will have to be incorporated into the IVIVC model. In such a case, the intercept of an in vitro-in vivo plot may not be zero. For the correlations illustrated in Figures 7 and 8, the IVIVC using the acid buffer dissolution profiles was superior to that obtained from water based on the statistical analysis of the line of best fit.

The predictability of the IVIVC is then determined by the validation procedure. Internal validation applies the correlation to the data used to develop the correlation. External validation applies the correlation to an independent data set not used to develop the correlation. Percent prediction errors (\%PE) are determined between predicted and observed values for $A U C$ and $C_{\max }$, and if these $\%$ PE values meet the FDA guidance criteria, validation of the IVIVC is complete. For the correlations illustrated in Figures 6 and 7, each correlation met both the internal and external validation criteria as shown in Tables 1 and 2, respectively. However, the \%PE for the internal validation for the correlation using water as the dissolution medium was marginally acceptable with a $15.0 \%$ PE for $C_{\max }$ for the Slow formulation.

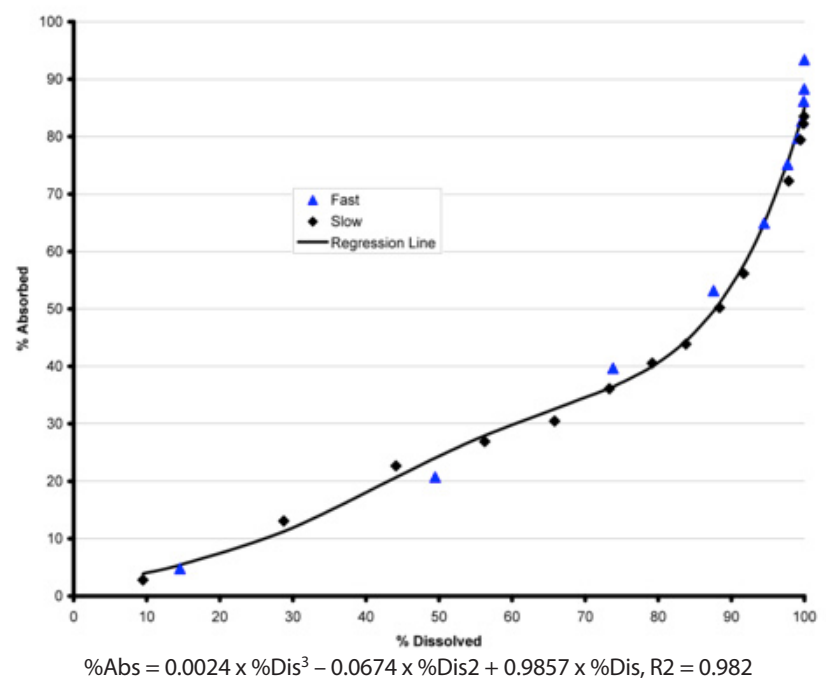

Figure 6. IVIVC attempt using water as dissolution medium for the slow and fast formulations.

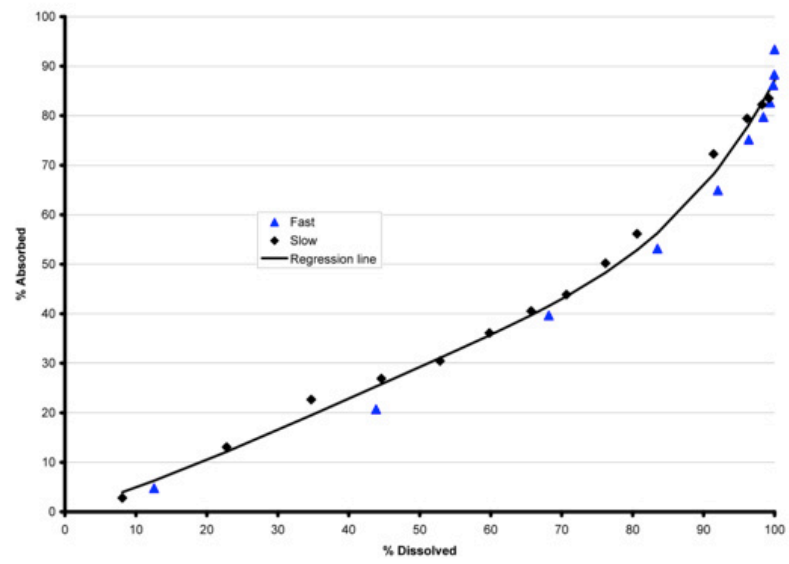

$\%$ Abs $=0.0001 \times \%$ Dis $^{3}+0.0016 \times \%$ Dis2 $+0.4687 \times \%$ Dis, R2 $=0.991$

Figure 7. IVIVC attempt using a pH 4.5 buffer as dissolution medium for the slow and fast formulations.

If the in vitro dissolution evaluation applied to a modified-release dosage form indicates that dissolution behavior is independent of the variables studied, then a Level A correlation is likely to exist. That is, when the in vitro dissolution curves are comparable to the drug absorption profiles, it is likely that a good correlation is present. If the dosage form exhibits dissolution behavior that varies with the in vitro conditions, then it must be determined which set of dissolution conditions best correlates with the in vivo drug release. It is important, however, to verify that the correlation is real and not an artifact. This 
may be achieved by preparing at least two formulations having significantly different in vitro dissolution rates. One should demonstrate a more rapid release and the other a slower release than the biobatch (3). A pilot BA-BE study should be performed with these formulations, and the previously established correlation demonstrated for both. The formulation modifications of these batches should be based on formulation factors that would be expected to influence the product's modified-release mechanism, and modification of these formulation factors are expected to influence the dosage form release rate.

Table 1. IVIVC Validation-Water

\begin{tabular}{|ccccccc|}
\hline \multicolumn{7}{|c|}{ Internal Predictability } \\
\hline \multirow{2}{*}{ Batch } & Obs & Pred & Pred & Obs & Pred & Pred \\
& AUC & AUC & Error & $\boldsymbol{C}_{\max }$ & $\boldsymbol{C}_{\max }$ & Error \\
\hline Slow & 38.0 & 38.0 & $0.14 \%$ & 1.13 & 1.30 & $15.0 \%$ \\
\hline Fast & 44.5 & 44.6 & $0.36 \%$ & 2.36 & 2.41 & $2.17 \%$ \\
\hline \multicolumn{7}{c|}{ Average } \\
$\%$ PE & $0.25 \%$ & Average & $8.59 \%$ \\
\hline \multicolumn{7}{c|}{ External Predictability } \\
\hline \multirow{2}{*}{ Batch } & Obs & Pred & Pred & Obs & Pred & Pred \\
& AUC & AUC & Error & $\boldsymbol{C}_{\max }$ & $\boldsymbol{C}_{\max }$ & Error \\
\hline Medium & 39.9 & 40.5 & $1.69 \%$ & 1.50 & 1.57 & $5.22 \%$ \\
\hline
\end{tabular}

Table 2. IVIVC Validation—Acetate Buffer

\begin{tabular}{|c|c|c|c|c|c|c|}
\hline \multicolumn{7}{|c|}{ Internal Predictability } \\
\hline Batch & $\begin{array}{l}\text { Obs } \\
\text { AUC }\end{array}$ & $\begin{array}{l}\text { Pred } \\
\text { AUC }\end{array}$ & $\begin{array}{l}\text { Pred } \\
\text { Error }\end{array}$ & $\begin{array}{l}\text { Obs } \\
C_{\max }\end{array}$ & $\begin{array}{l}\text { Pred } \\
C_{\max }\end{array}$ & $\begin{array}{l}\text { Pred } \\
\text { Error }\end{array}$ \\
\hline Slow & 38.0 & 38.8 & $2.06 \%$ & 1.13 & 1.17 & $3.63 \%$ \\
\hline Fast & 44.5 & 44.9 & $1.06 \%$ & 2.36 & 2.46 & $4.54 \%$ \\
\hline & \multicolumn{2}{|c|}{$\begin{array}{c}\text { Average } \\
\% \mathrm{PE}\end{array}$} & $1.56 \%$ & \multicolumn{2}{|c|}{$\begin{array}{c}\text { Average } \\
\% \mathrm{PE}\end{array}$} & $4.09 \%$ \\
\hline \multicolumn{7}{|c|}{ External Predictability } \\
\hline Batch & $\begin{array}{l}\text { Obs } \\
\text { AUC }\end{array}$ & $\begin{array}{l}\text { Pred } \\
\text { AUC }\end{array}$ & $\begin{array}{l}\text { Pred } \\
\text { Error }\end{array}$ & $\begin{array}{l}\text { Obs } \\
C_{\max }\end{array}$ & $\begin{array}{l}\text { Pred } \\
C_{\max }\end{array}$ & $\begin{array}{l}\text { Pred } \\
\text { Error }\end{array}$ \\
\hline Medium & 39.9 & 40.0 & $0.46 \%$ & 1.50 & 1.62 & $8.36 \%$ \\
\hline
\end{tabular}

Alternatively, the in vivo performance of the biobatch formulation can be simulated based upon the correlation developed with the formulations that were used in the BA-BE study. A comparison of the predicted and experimentally determined values can then be made as illustrated in Figures 8 and 9. The dissolution profiles of the formulations are applied to the IVIVC model to predict the in vivo input profile. These in vivo input profiles are convoluted to simulate the in vivo plasma concentrationtime profiles. Thus, the exercise illustrated in Figures 8 and 9 serves as an internal validation of the Level A correlation.
An external validation simulates data for a formulation batch that was not included in the Level A correlation calculations. Such a validation was performed using the in vivo data from the Medium lot of the formulation, and the results are illustrated in Figure 10.

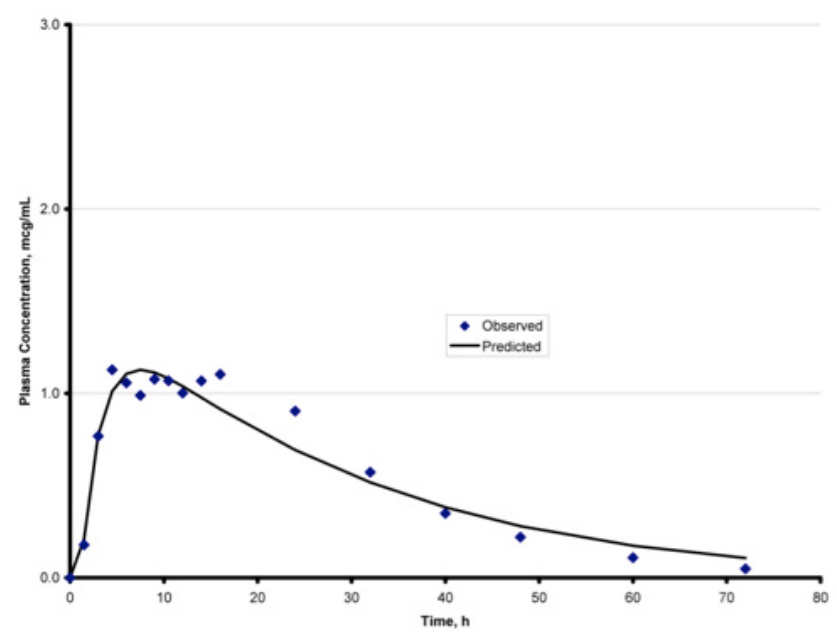

Figure 8. Observed and predicted mean plasma profiles: slow formulation.

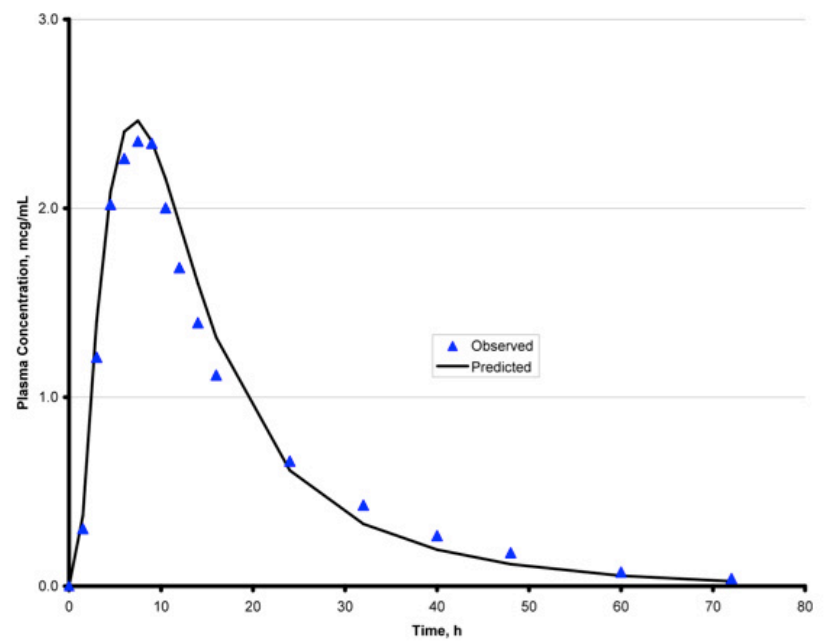

Figure 9. Observed and predicted mean plasma profiles: fast formulation.

Once a Level A correlation is established, in vitro testing may be utilized for establishing dissolution specifications. Traditionally, a multipoint dissolution specification for a modified-release dosage form has been based on the in vitro dissolution of the biobatch as well as a manufacturing history. By evaluating the dissolution of several lots besides the biobatch, a dissolution specification could be proposed with a range of $\pm 2.5-3$ standard deviations for each dissolution time point. The difficult decision is how much variation should be allowed around each time point; that defines the extremes of the dissolution specification. While a manufacturer might prefer wider specifications, a regulatory agency would prefer more narrow limits to the specifications. When a Level A correlation has been established, the dissolution specifications may be defined and supported by either convolution or deconvolution. 


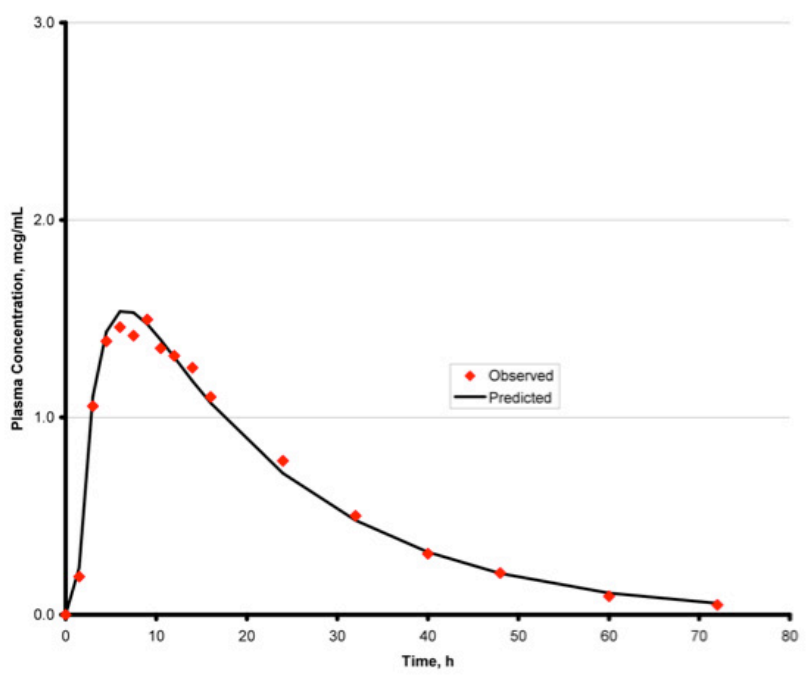

Figure 10. Observed and predicted mean plasma profiles: medium formulation.

To utilize convolution, desirable upper and lower dissolution values can be selected for each time point established from the biobatch, and the average dissolution values will be approximately the same as those of the biobatch. The dissolution curves defined by the upper and lower extremes are applied to the IVIVC model to generate predicted in vivo input profiles. These in vivo input profiles are convoluted to predict the anticipated plasma level curves that would result from administration of these formulations to a group of human volunteers. If the resulting plasma level data fall within the $95 \%$ confidence intervals obtained in the definitive BA-BE study, the dissolution specification ranges should be acceptable.

In a deconvolution approach, a set of plasma-level data is established both for a hypothetical formulation with a more rapid release than that of the biobatch and for one demonstrating a slower release. These may be selected by using the extremes of the $95 \%$ confidence intervals or by selecting high and low values around the mean plasma profile ( \pm 1 S.D.). These curves are then deconvoluted, and the resulting input rate curve is used to establish the upper and lower dissolution specifications at each time point.

In the case of Level B and C correlations, batches of product must be made at the proposed upper and lower limits of the dissolution range, and it must be demonstrated that these batches are acceptable by performing a BA-BE study.

\section{COMPUTER SOFTWARE FOR DEVELOPMENT, VALIDATION, AND APPLICATION OF IVIVC}

There is a considerable amount of data, and data manipulation, to process in establishing an IVIVC. Therefore, it is highly recommended that a validated software package be used. Three packages that are available for use are:

- IVIVC Toolkit for Phoenix WinNonlin from Certara.

- Simcyp from Certara.

- GastroPlus from Simulations Plus, Inc.

\section{CONCLUSIONS}

The benefits of a Level A IVIVC are readily apparent when one considers the time and expense of conducting bioavailability studies to demonstrate bioequivalence between a SUPAC formulation and the marketed formulation. The establishment of an IVIVC also provides benefits during the development of the drug product. The development of a biorelevant dissolution method can increase the confidence in the development process, since the formulation scientist will know which formulation, manufacturing, and process parameters will affect the in vivo performance of the product being developed. In addition, if a biowaiver is granted on the basis of a Level A IVIVC, the number of BA/BE studies required during the development of a new formulation may be greatly reduced. As previously discussed, support for establishing dissolution specifications and SUPAC are further advantages of establishing a Level A IVIVC. It has been questionable whether such an extrapolation with Level $B$ and $C$ correlations is possible, but the example of a Level C presented in the CRS Workshop (9) suggests that with a strong correlation, any Level may lead to success. It must be realized, however, that an IVIVC is unique to each drug and drug product, and it is recognized that it is not possible to develop an IVIVC in all cases. As Dr. Marroum presented in the 2013 CRS Workshop dealing with IVIVC, most of the formulations with successful IVIVC models had been primarily extended-release formulations including controlled-release injectables, implants, vaginal rings, and drug-eluting stents. IVIVC failures were primarily immediate-release products and transdermal drug delivery systems, and the authors concur with his assessment. His presentation also helped to demonstrate that regulatory agencies are open to creative attempts at establishing in vitro-in vivo correlations.

\section{REFERENCES}

1. Wagner, J. G.; Nelson, E. Per Cent Absorbed Time Plots Derived from Blood Level and/or Urinary Excretion Data. J. Pharm. Sci. 1963, 52 (6), 610-611. DOI: 10.1002/ jps.2600520629.

2. Levy, G.; Leonards, J. R.; Procknal, J. A. Development of In Vitro Dissolution Tests Which Correlate Quantitatively with Dissolution Rate-Limited Drug Absorption in Man. J. Pharm. Sci. 1965, 54 (12), 1719-1722. DOI: 10.1002/ jps.2600541204.

3. Gonzalez, M. A.; Golub, A. L. THEO-DUR and THEODUR Sprinkle ${ }^{\mathrm{TM}}$ : Controlled-release delivery systems for theophylline. Drug Dev. Ind. Pharm. 1983, 9 (7), 13791396. DOI: $10.3109 / 03639048309046324$.

4. Loo, J. C. K.; Riegelman, S. New Method for Calculating the Intrinsic Absorption Rate of Drugs. J. Pharm. Sci. 1968, 57 (6), 918-928. DOI: 10.1002/jps.2600570602.

5. Wagner, J. G. Pharmacokinetic Absorption Plots from Oral Data Alone or Oral/Intravenous Data and An Exact Loo-Riegelman Equation. J. Pharm. Sci. 1983, 72 (7), 
838-842. DOI: 10.1002/jps.2600720738.

6. Extended Release Oral Dosage Forms: Development, Evaluation, and Application of In Vitro/In Vivo Correlations; Guidance for Industry; U.S. Department of Health and Human Services, Food and Drug Administration, Center for Drug Evaluation and Research (CDER), U.S. Government Printing Office: Washington, DC, 1997.

7. USP Subcommittee on Biopharmaceutics. In Vitro/ In Vivo Correlation for Extended Release Oral Dosage Forms. Pharm. Forum 1988, 14 (4), 4160-4161.

8. <1088> In Vitro and In Vivo Evaluation of Dosage Forms. In The United States Pharmacopeia and National Formulary USP 36-NF 31; The United States Pharmacopeial Convention, Inc.: Rockville, MD, 2013. http://www.pharmacopeia.cn/v29240/usp29nf24s0_ c1088.html (accessed April 1, 2015).

9. Gonzalez, M. A.; Gray, V. A. CRS Workshop Report: Using an In Vitro-In Vivo Correlation (IVIVC) to Meet Challenges in Global Delivery. Dissolution Technol. 2013, 20 (4), 42-44. DOI: 10.14227/DT200413P42. 